\title{
EFECTO DEL ÁCIDO NAFTALENACÉTICO, EN EL ENRAIZAMIENTO DE ESTACAS DE Myrciaria dubia (HBK) MC VAUGH, CAMU CAMU
}

\author{
EFFECTS OF NATALENACETIC ACID, IN THE STAKE - ROOTING \\ OF Myrciaria dubia (HBK) MC VAUGH, CAMU CAMU
}

\author{
Carlos Oliva ${ }^{1}$ y Antonio López ${ }^{2}$
}

\section{RESUMEN}

Con el objetivo de mejorar la propagación asexual del camu camu, se instaló en la Estación Experimental del IIAP Ucayali, un ensayo bajo un diseño de bloques completos al azar con arreglo factorial (4x2) con tres repeticiones. Los factores fueron cuatro dosis de ANA (0, 100, 200 y 300 ppm) y dos tiempos de inmersión (30 y 60 minutos). Las estacas tuvieron $25 \mathrm{~cm}$ de longitud y diámetro entre 0,8 y $1 \mathrm{~cm}$, provenientes de las plantas establecidas en la Estación, con riegos 2 veces/día (2 minutos/riego). Fueron evaluados: el porcentaje de brotación a los 60 y 90 días, y el porcentaje de enraizamiento y callos a los 120 días. El mayor porcentaje de enraizamiento (24.47\%) se obtuvo con la combinación de 200 ppm ANA y 30 minutos de inmersión. Encontramos diferencias significativas (Duncan $=0.05$ ) entre dosis de ANA, para 100 ppm de ANA con 15.57\% en enraizamiento y 65.56\% en formación de callos en el tratamiento 00 ppm de ANA; no se encontraron diferencias entre tiempos de inmersión. También se encontraron diferencias significativas entre la interacción dosis por tiempo de inmersión para enraizamiento, considerando como la mejor combinación 100 ppm de ANA con 30 minutos de inmersión con 24.47\% de enraizamiento.

Palabras clave: camu camu, estaca, enraizamiento e inmersión.

\section{ABSTRACT}

With the objective of improving the asexual propagation of the camu camu, it settled in the Experimental Station of the IIAP Ucayali, a low rehearsal a design of complete blocks at random with factorial arrangement (4x2) with three repetitions. The factors were 4 dose of $\operatorname{ANA}(0,100,200$ and $300 \mathrm{ppm})$ and two times of immersion (30 and 60 minutes). The stakes had $25 \mathrm{~cm}$ longitude and diameter among 0,8 to $1 \mathrm{~cm}$, coming from the plants settled down in the Station, with waterings 2 veces/día ( 2 minutos/riego). They were evaluated the brotación percentage to the 60 and 90 days, and the enraizamiento percentage and tripes to the 120 days. The biggest percentage of enraizamiento (24.47)\% was obtained with the combination of 200 ppm ANA and 30 minutes of immersion. We find significant differences (Duncan $=0.05$ ) among dose of ANA, for 100 ppm of ANA with $15.57 \%$ in enraizamiento and $65.56 \%$ in formation of tripes in the treatment 00 ppm of ANA, he/she was not differences among times of immersion. It was also significant differences among the interaction dose * time of immersion for enraizamiento, whereas clause as the best combination 100 ppm of ANA with 30 minutes of immersion with 24.47 enraizamiento\%.

Key words: camu camu, stakes, rooting, immersion, myrciaria, nafta lenacenc acid.

\section{INTRODUCCIÓN}

El Myrciaria dubia HBK Mc Vaugh, Myrtaceae, camu camu, es una fruta nativa de la Amazonía, arbusto de 3 a 8 metros, cuyo hábitat son los márgenes de los ríos y lagos en afluentes de agua negra del río Amazonas. Es la especie con más alto contenido de ácido ascórbico en el mundo.

En la propagación vegetativa de esta especie, se ha desarrollado el método de injertación por astillas. Enciso (1992), obtuvo prendimientos de $83.3 \%$. Sin embargo, el método no es el más adecuado cuando deseamos incursionar en el mejoramiento genético por la influencia del «patrón» sobre la calidad genética o la expresión

1 Investigador, Instituto de Investigaciones de la Amazonía Peruana coliva@iiap.org.pe - cel. 9907869

2 Investigador IIAP Ucayali jlopez@iiap.org.pe 
fenotípica del «injerto yema». En efecto en la propagación por estacas se reproduce por medio de la réplica del ADN toda la información genética de la planta madre (Vásquez 2000).

Hudson \& Dale (1995), sustentan que la propagación por estaca consiste en provocar el enraizamiento y la brotación de un trozo de tallo, raíz u hojas separadas de la planta madre.

Según Hartmann (1995), existen factores que determinan la capacidad de enraizamiento de las estacas, considerando que el incremento de la edad reduce la capacidad de formar raíces adventicias. Lorente (1999), informa que para favorecer y aumentar el enraizamiento de las estacas, se puede recurrir a una serie de técnicas, tales como: aplicación de hormonas, nebulización, humidificación y calentamiento basal.

Rodrigues \& Ono (1996), relatan que la formación de callos se efectúa generalmente antes de la iniciación y del desenvolvimiento de las raíces y raramente la formación de raíces es directamente del callo. También Carrera (1977), citado por Silva (1985), dice que el callo es una formación regenerativa que ocurre principalmente por el estímulo de la actividad cambial y por eso no siempre está relacionado con la formación de raíces.

Utia y Pinedo (1979), ensayó el enraizamiento con dos tipos de estacas de camu camu, apicales de $0.5 \mathrm{~cm}$ de diámetro y estacas basales de $0.5-1 \mathrm{~cm}$ de diámetro. Sometidos a tres tipos de sustratos: arena, tierra y aserrín; en ninguno de los casos se obtuvo enraizamiento. Así mismo, Baos et al. (1987), opinaron que la propagación vegetativa del camu camu, presenta dificultades para su enraizamiento.

Vásquez (2000), efectuó enraizamiento de estacas de camu camu, utilizando tres tipos de diámetro (0,5; 2,0 y mayor de 2,5 cm), sembrados en posición inclinada, con riegos diarios. Los resultados fueron 0, 10 y 20\% de enraizamiento para los diámetros 0,$5 ; 2,0$ y $>2,5 \mathrm{~cm}$ respectivamente.

Galucio, P.B. (2002), utilizando estacas con diámetros mayores que 0,8 mm de diámetro y aplicando 200 ppm de ácido naftalenacético (ANA), logró a los 90 días hasta 90\% de enraizamiento.

El presente estudio, tuvo como objetivo evaluar el enraizamiento de esquejes de camu camu, utilizando 00, 100, 200 y 300 ppm de ácido naftalenacético y dos tiempos de inmersión.

\section{MATERIAL Y MÉTODO}

Entre los meses de febrero a junio de 2003, se desarrolló el ensayo empleando estacas de $25 \mathrm{~cm}$ de longitud y diámetro de 0.8 a $1.0 \mathrm{~cm}$. El material fue colectado de plantas con siete años de edad, localizadas en la Estación Experimental del IIAP Ucayali. Su localización esta en las coordenadas geográficas de $8^{\circ} 22^{\prime} 31^{\prime \prime}$ de latitud sur, $74^{\circ}$ 34' 35" de longitud oeste, a una altitud de 154 msnm.

Las plantas están establecidas, en las instalaciones de la EE-IIAP Ucayali, con suelos de textura arcillosa; muy ácida con pH 4.6; deficiente concentración de fósforo, potasio y boro, toxicidad de aluminio, y normal contenido de materia orgánica, con CIC 13.25 meq/100 g de suelo (López 2002). La zona presenta una temperatura promedio máxima de $34.4^{\circ} \mathrm{C}$, una mínima de $20.2^{\circ} \mathrm{C}$, y una humedad relativa de $82.93 \%$.

Los tratamientos se distribuyeron bajo un diseño de bloque completo aleatorizado (BCA) DBCA con arreglo factorial 4x2, con tres repeticiones. La unidad experimental o parcelas contaron con 15 estacas. Los niveles para el factor dosis de ANA fueron 0,100, 200 y 300 ppm Los niveles para tiempo de inmersión 30 y 60 minutos, totalizando ocho tratamientos, con 15 estacas/repetición y 45 por tratamiento. Las estacas fueron establecidas en camas (1 x $3 \mathrm{~m}$ ) conteniendo arena como sustrato, cubiertas con hojas de hirapay, con irrigación de aspersor nebulizado 2 veces/día (2 minutos/riego). 
Las evaluaciones fueron realizadas a los 60, 90 y 120 días después de la instalación del experimento. Los parámetros evaluados fueron: porcentaje de brotación a los 60 y 90 días y porcentaje de enraizamiento y formación de callos a los 120 días.

\section{RESULTADOS Y DISCUSIÓN}

A sesenta días después de la instalación, el brotamiento foliar es eficiente en todo los tratamientos, superando fácilmente el 60\%; el tratamiento testigo ha logrado mejores resultados hasta 97,67\% de brotación en 60 minutos de inmersión, seguido por 200 ppm ANA con 30 minutos de inmersión con 95.67\%. Sin embargo a los 90 días el porcentaje de brotamiento se reduce en cada tratamiento, esto podría ocurrir debido al agotamiento de reservas nutricionales en las estacas; al no lograr la emisión de raíces, las yemas se secan y mueren paulatinamente (tabla 1).

Tabla 1. Comportamiento promedio porcentual de enraizamiento y formación de callos en esquejes de camu camu arbustivo, con la aplicación de tres dosis de ácido naftalenacético y dos tiempos de inmersión.

\begin{tabular}{cccc}
\hline \multirow{2}{*}{ Dosis de ANA } & Tiempo & \multicolumn{2}{c}{ Porcentaje de brotamiento } \\
\cline { 2 - 4 } & inmersión & $\mathbf{6 0}$ días & $\mathbf{9 0}$ días \\
\hline \multirow{2}{*}{$000 \mathrm{ppm}$} & $30 \mathrm{~min}$. & 82.33 & 77.77 \\
& $60 \mathrm{~min}$. & 97.67 & 80.00 \\
\multirow{2}{*}{$100 \mathrm{ppm}$} & $30 \mathrm{~min}$. & 66.67 & 64.43 \\
& $60 \mathrm{~min}$. & 82.33 & 62.23 \\
\multirow{2}{*}{$200 \mathrm{ppm}$} & $30 \mathrm{~min}$. & 95.67 & 93.30 \\
& $60 \mathrm{~min}$. & 73.67 & 66.63 \\
\multirow{2}{*}{$300 \mathrm{ppm}$} & $30 \mathrm{~min}$. & 73.33 & 71.10 \\
& $60 \mathrm{~min}$. & 91.00 & 84.47 \\
\hline
\end{tabular}

El brotamiento en estacas de camu camu no es preocupante para la propagación, el problema es la formación de raíces, que va a permitir absorber los nutrientes y alimentar a los brotes, constituyéndose de esta manera en una nueva planta.

El enraizamiento fue mejor con 100 ppm de ANA en 30 minutos de inmersión con 24.46\% de enraizamiento; con esta misma dosis y con 60 minutos se logró $6.67 \%$ de enraizamiento, similar al comportamiento del tratamiento testigo. Cuando la dosis de ANA se incrementa, el porcentaje de enraizamiento es menor, esto se refleja en los dos tiempos de inmersión, siendo mayor en 60 minutos de inmersión llegando a 0.00\% de enraizamiento con 300 ppm de ANA (tabla 2).

Con 200 ppm de ANA, se logró hasta 6.7\% de enraizamiento en 30 minutos de inmersión y fue menor cuando el tiempo se inmersión se incrementó, resultado que contradice al reportado por Galucio (2002), quien logró hasta 90\% de enraizamiento utilizando 200 ppm de ANA con diámetros mayores a $0.8 \mathrm{~cm}$. 
Tabla 2: Comportamiento porcentual del enraizamiento y formación de callos de estacas de camu camu, mediante la aplicación de ácido naftalenacético en dos tiempos de inmersión.

Variable dependiente: Enraizamiento

\begin{tabular}{llrcr}
\hline & $\begin{array}{c}\text { Dosis } \\
\text { ANA }\end{array}$ & 30 min. & $\begin{array}{c}\text { Tiempos de inmersión } \\
\mathbf{6 0 ~} \mathbf{~ m i n} .\end{array}$ & Total \\
\hline \% enraizamiento & $000 \mathrm{ppm}$ & 4.467 & 6.700 & 5.583 \\
de estacas & $100 \mathrm{ppm}$ & 24.467 & 6.667 & 15.567 \\
& $200 \mathrm{ppm}$ & 6.700 & 4.433 & 5.567 \\
& $300 \mathrm{ppm}$ & 2.333 & .000 & 1.117 \\
& Total & 9.467 & 4.450 & 6.958 \\
\hline \% formación de & $000 \mathrm{ppm}$ & 68.89 & 62.22 & 65.56 \\
& $100 \mathrm{ppm}$ & 40.00 & 31.11 & 35.56 \\
& $200 \mathrm{ppm}$ & 37.78 & 24.44 & 31.11 \\
& $300 \mathrm{ppm}$ & 20.00 & 20.00 & 20.00 \\
& Total & 41.67 & 34.44 & 38.06 \\
\hline
\end{tabular}

En la formación de callos, observamos (tabla 2), que el tratamiento testigo (00 ppm de ANA) logró los mejores resultados con 68.89 y 62.22 \% para 30 y 60 minutos de inmersión respectivamente. Además, es importante observar que la aplicación creciente de dosis de ANA disminuye el porcentaje de formación de callos. Estos resultados concuerdan con lo expuesto por Carrera García (1977), que el callo es una formación regenerativa y no siempre está ligado con la formación de raíces una vez que son procesos fisiológicos independientes, a esto complementa Rodríguez \& Ono (1996), que la formación del callo se efectúa generalmente antes de la iniciación y desenvolvimiento de las raíces, pero las raíces muy raramente con formadas de callos.

Se encontraron diferencias significativas en porcentaje de enraizamiento y formación de callos entre tratamientos, dosis e interacción dosis x tiempo, pero no entre tiempos de inmersión (tablas 3 y 4). La mejor dosis fue 100 ppm de ANA con $15.57 \%$ de enraizamiento y las dosis 000 ppm, 200 ppm y 300 ppm se comportaron estadísticamente similares. En relación al porcentaje de callos, fue mejor el testigo con $65.56 \%$ de callos (tabla 3), resultado que no concuerda con Galucio (2002), quien encontró hasta 90\% de enraizamiento en solución de 200 ppm de ANA con diámetros mayores de $0.8 \mathrm{~cm}$. Probablemente una de las diferencias sea debido al empleo de diámetros, esto corrobora con lo reportado por Vásquez (2000) quien logró mejores resultados utilizando diámetros mayores de $2.5 \mathrm{~cm}$.

Tabla 3. Nivel de callogensis y enraizamiento bajo la influencia de ANA (prueba de medias Duncan a = 0,05).

\begin{tabular}{lcc}
\hline Dosis de ANA & \% de enraizamiento & \% formación de callos \\
\hline $000 \mathrm{ppm}$ & $5.58 \mathrm{~b}$ & $65.56 \mathrm{a}$ \\
$100 \mathrm{ppm}$ & $15.57 \mathrm{a}$ & $35.56 \mathrm{~b}$ \\
$200 \mathrm{ppm}$ & $5.57 \mathrm{~b}$ & $31.11 \mathrm{~b}$ \\
$300 \mathrm{ppm}$ & $1.12 \mathrm{~b}$ & $20.00 \mathrm{~b}$ \\
\hline $30 \mathrm{~min}$ & $9.47 \mathrm{a}$ & $41.67 \mathrm{a}$ \\
$60 \mathrm{~min}$ & $4.45 \mathrm{a}$ & $34.44 \mathrm{a}$ \\
\hline
\end{tabular}

Las letras representan la prueba estadística de Duncan $=0.05$. 
En la interacción dosis x tiempo de inmersión (tabla 4), la mejor combinación fue 100 ppm ANA en 30 minutos de inmersión con $24.47 \%$ de enraizamiento, resultado significativamente mejor que las demás combinaciones. Con 300 ppm de ANA y 60 minutos de inmersión se obtuvo cero porciento de enraizamiento, de lo que se podría inferir que a mayor dosis de ANA y mayor tiempo de inmersión la probabilidad de enraizamiento es menor.

Tabla 4. Porcentaje de enraizamiento bajo la influencia de ANA y dos tiempos de inmersión (prueba de medias Duncan $\mathrm{a}=0,05)$.

\begin{tabular}{|c|c|c|c|}
\hline \multirow[b]{2}{*}{ Dosis vs Tiempo } & \multirow[b]{2}{*}{$\mathrm{N}$} & \multicolumn{2}{|c|}{ Subconjunto } \\
\hline & & 1 & 2 \\
\hline 300 ppm. 60 min. & 3 & .000 & \\
\hline 300 ppm. 30 min. & 3 & 2.233 & \\
\hline 200 ppm. 60 min. & 3 & 4.433 & \\
\hline 000 ppm. 30 min. & 3 & 4.467 & \\
\hline 100 ppm. 60 min. & 3 & 6.667 & \\
\hline 000 ppm. 60 min. & 3 & 6.700 & \\
\hline 200 ppm. 30 min. & 3 & 6.700 & \\
\hline 100 ppm. 30 min. & 3 & & 24.467 \\
\hline Sig. & & .321 & 1.000 \\
\hline
\end{tabular}

a. Uses Harmonic Mean Sample Size $=3.000$.

b. Alpha $=.05$.

En relación a la formación de callos (tabla 5), según la prueba de Duncan (0.05), se observaron tres niveles de distribución, considerando como la mejor combinación 000 ppm de ANA con 30 minutos de inmersión, quien se comporta similar a 000 ppm con 60 minutos de inmersión pero significativamente mejor a las demás combinaciones. Lo que nos quiere decir que las estacas de camu camu sin aplicación de hormonas tiene mayor formación de callos.

Tabla 5. Porcentaje de callos bajo la influencia de ANA y dos tiempos de inmersión (prueba de medias Duncan $\mathrm{a}=0,05)$.

\begin{tabular}{|c|c|c|c|c|}
\hline \multirow[b]{2}{*}{ Dosis vs Tiempo } & \multirow[b]{2}{*}{$\mathrm{N}$} & \multicolumn{3}{|c|}{ Subconjunto } \\
\hline & & 1 & 2 & 3 \\
\hline 300 ppm. 30 min. & 3 & 20.00 & & \\
\hline 300 ppm. 60 min. & 3 & 20.00 & & \\
\hline 200 ppm. 60 min. & 3 & 24.44 & & \\
\hline 100 ppm. 60 min. & 3 & 31.11 & & \\
\hline 200 ppm. 30 min. & 3 & 37.78 & 37.78 & \\
\hline 100 ppm. 30 min. & 3 & 40.00 & 40.00 & \\
\hline 000 ppm. 60 min. & 3 & & 62.22 & 62.22 \\
\hline 000 ppm. 30 min. & 3 & & & 68.89 \\
\hline Sig. & & 180 & .089 & .609 \\
\hline
\end{tabular}

a. Uses Harmonic Mean Sample Size $=3.000$.

b. Alpha $=.05$. 


\section{CONCLUSIONES}

- El brotamiento foliar en estacas de camu camu, se mostró alto en todos los tratamientos y combinaciones.

- El porcentaje de enraizamiento de estacas fue mayor en la combinación de 100 ppm de ácido naftalenacético (ANA) con 30 minutos de inmersión.

- La formación de callos es mayor cuando los esquejes son tratados a 00 ppm de hormona enraizante (ANA).

\section{RECOMENDACIONES}

- Se recomienda realizar trabajos con otras sustancias enraizantes y emplear otras dosis y otros tiempos de inmersión.

- Se recomienda utilizar este experimento en el enraizamiento de estacas con diámetros mayores de un centimetro, porque a mayor diámetro existen mayores reservas y por ende mayor probabilidad de enraizamiento.

- Se deben proteger las macas de enraizado, para subir la temperatura interior y acelerar los procesos químicos y por ende la formación de raíces.

- Es recomendable tomar en cuenta el tiempo de riego, probablemente esto sea uno de los factores que haya determinado el bajo porcentaje de enraizamiento de esquejes.

- Se deben considerar plantas sanas y muy bien fertilizadas para extraer las estacas.

\section{BIBLIOGRAFÍA}

BAOS, C.P.; B.F.D. FLOR y C.P. TRUEBA. 1986. Descriptores de camu camu. Programa Nacional de Cultivos Tropicales. (Informe Técnico, n. ${ }^{\circ}$ 8), 55 p.

CALZADA, J. 1980. 143 frutales nativos. UNALM. Lima-Perú. 320 p.

ENCISO, R. 1992. Propagación de camu-camu (Myrciaria dubia) por injerto. Informe Técnico n. ${ }^{\circ} 18$. Programa de Investigación en Cultivos Tropicales-INIA. Lima. 17 Pág.

FLORES, P. S. 1997. Cultivo de Frutales Nativos Amazónicos. Manual para extensionistas. TCA. Lima-Perú. 307 p.

GALUCIO, P. B. 2002. Producción de mudas de camu camu (Myrciaria dubia (H.B.K.) Mc Vaugh) por estacas utilizando ramas provenientes de diferentes tipos y posiciones de la planta.Nota Técnica. INPA-Brasil.

HARTMANN y KESTER. 1995. Propagación de Plantas. Editorial Continental. México. 760 p.

HUDSON, T. H. y E. K. DALE. 1995. Propagación de plantas. Editorial Continental. México. 733 p.

LORENTE, H. J. 1999. Biblioteca de la Agricultura. Editorial LEXUS. Pág. 130-131.

LÓPEZ A.U. 2002. Aplicación de niveles de calcio, boro, cobre y zinc, sobre la productividad del camu camu en suelos aluviales. Informe Trimestral 2003 IIAP Ucayali. 
MARCIO, P. 2003. Propagación vía estacas apicales, caracterización morfológica y molecular de Jabuticabeiras (Myrciaria spp.). Tesis presentada a la Escuela Superior de Agricultura para obtener el título de doctorado, Universidad de Sao Paulo-Brasil.

RODRIGUES, J.D.; y E.O. ONO. 1996. «Aspectos de la fisiología del enraizamiento de estacas caulinares. Jaboticabal: FUNEP, 83 p.

SILVA, I.C. (1985) Propagación Vegetativa; Aspectos Morfológicos. Itabuna: CEPLAC. V 4, P 1-26 (boletín Técnico- Brasil.

UTIA Y PINEDO (1979), Propagación del Arazá y camu camu. Tésis presentada para optar el titulo de Ingeniero Agrónomo. Universidad Nacional de la Amazonia Peruana. Iquitos-Perú.

VÁSQUEZ MATUTE A. (2000) «El Camu camu, Cultivo, Manejo e Investigaciones». Universidad Nacional de la Amazonía Peruana- Facultad de Agronomía- Iquitos-Perú. 